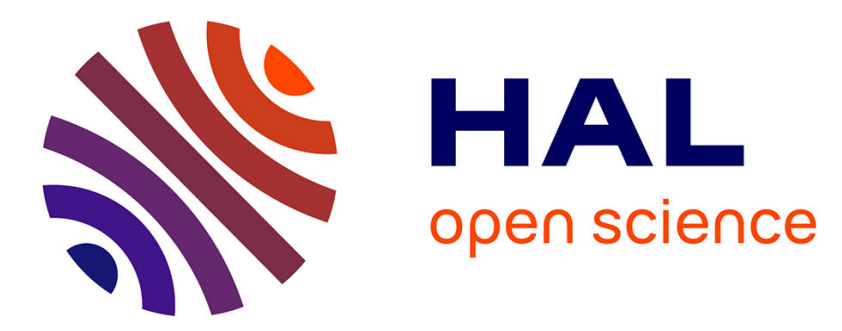

\title{
CHAPTER 8. Sustainable Activation of Chemical Substrates Under Sonochemical Conditions
}

Micheline Draye, Marion L. Chevallier, Vanille Quinty, Claire Besnard, Alexandre Vandeponseele, Gregory Chatel

\section{To cite this version:}

Micheline Draye, Marion L. Chevallier, Vanille Quinty, Claire Besnard, Alexandre Vandeponseele, et al. CHAPTER 8. Sustainable Activation of Chemical Substrates Under Sonochemical Conditions. Sustainable Organic Synthesis: Tools and Strategies, Royal Society of Chemistry, pp.212-238, 2021, Sustainable Organic Synthesis: Tools and Strategies, 10.1039/9781839164842-00212 . hal-03546738

\author{
HAL Id: hal-03546738 \\ https://hal.science/hal-03546738
}

Submitted on 28 Jan 2022

HAL is a multi-disciplinary open access archive for the deposit and dissemination of scientific research documents, whether they are published or not. The documents may come from teaching and research institutions in France or abroad, or from public or private research centers.
L'archive ouverte pluridisciplinaire $\mathbf{H A L}$, est destinée au dépôt et à la diffusion de documents scientifiques de niveau recherche, publiés ou non, émanant des établissements d'enseignement et de recherche français ou étrangers, des laboratoires publics ou privés. 
1 X. Sustainable Activation of Chemical Substrates Under Sonochemical

2 Conditions

3

4

5

6

7

8 M. Draye, M. Chevallier, ${ }^{a}$ V. Quinty, ${ }^{a}$ C. Besnard, ${ }^{a}$ A. Vandeponseele ${ }^{a}$ and G.

9 Chatel ${ }^{\mathrm{a}^{*}}$.

a Univ. Savoie Mont Blanc, CNRS, EDYTEM, 73000 Chambéry, France.

*corresponding email address: gregory.chatel@univ-smb.fr

\section{ABSTRACT}

14 Ultrasound activation has been widely explored in organic chemistry to improve the 15 yields of reaction or their kinetics. The sonochemical approach can also allow 16 changing the selectivities or limiting the use of hazardous solvents or the amount of 17 catalyst, in accordance with the principle of green chemistry. However, a rigorous 18 control of the sonochemical parameters is necessary to better understand the 19 mechanisms involved under ultrasonic activation. 


\section{1. Introduction}

Sonochemistry is the use of power ultrasound for chemical reactions. Some chemists reported the first reaction involving ultrasound in $1927,{ }^{1,2}$ although the term "sonochemistry" has only been used since $1980 .^{3}$ Ultrasound is often viewed, and particularly in organic chemistry, as a simple and efficient mixing tool in the lab. However, a control of the sonochemical parameters and experimental conditions have made it possible to demonstrate, in many cases, significant improvements in terms of reaction yields and/or reaction speed, thanks to the use of ultrasound. In some cases, unexpected reactivities and selectivities have also been observed under ultrasound, making it possible to imagine new perspectives and applications of sonochemistry in organic chemistry. After having introduced sonochemistry by presenting its basic theoretical and practical aspects, some case studies from recent literature are summarized to show the major advantages of using ultrasound in an organic reaction. At last, this chapter aims to highlight the current and future challenges of organic sonochemistry.

\section{2. Sonochemistry, a chemistry based on power ultrasound}

\section{2. 1. Acoustic cavitation and associated effects}

Acoustic cavitation in liquid media is the phenomenon of formation, growth and violent collapse induced by sound waves that generate fluctuation of pressure. Liquid media usually contains free gas bubbles or gas molecules trapped in solid impurities, which can act as nuclei for cavitation ${ }^{4}$. Once the cavitation bubble is formed, its diameter increases throughout the expansion and compression phases to achieve a critical size at which it violently collapses. Indeed, expansion phases being isotherm and compression phases adiabatic, a large amount of acoustic energy is 
accumulated inside the bubble. At the moment of bubble implosion, temperatures of about $5,000 \mathrm{~K}$ and pressures close to 1,000 bar are then reached within the bubble (see Figure X.1). These extremes conditions lead to different local effects such as radicals' formation, shock waves, acoustic micro-currents and violent liquid microjets, which are at the origin of application of sonochemistry ${ }^{5,6}$.

$$
\text { [Insert Figure X.1 here] }
$$

\section{2. 2. Ultrasonic parameters and experimental factors affecting cavitation}

The ambient conditions of a reaction system can strongly influence acoustic cavitation threshold and its intensity, which then directly affects the kinetic and/or the yield of the chemical reaction. The acoustic cavitation in the liquid may be affected by several parameters such as the frequency and power of the ultrasounds, the hydrostatic and external pressures, the temperature, the nature of the solvent and dissolved gas.

\section{2. 2. 1. Ultrasonic frequency}

Ultrasound is a sound wave with a frequency (f, Eq. X.1) greater than the upper limit of human hearing, which is generally over $20 \mathrm{kHz}$ and below $10 \mathrm{MHz}$. When it propagates in an elastic medium it presents all the general properties of periodic progressive waves such as propagation, attenuation and reflection ${ }^{7}$.

[Insert Equation X.1 here]

In water low frequencies, ranging between 20 and $80 \mathrm{kHz}$, lead to relatively few and large transient cavitation bubbles; Physical effects such as micromixing, erosion, etc. predominate over chemical effects. High frequencies, ranging between 150 and 
2,000 kHz, produce many small transient cavitation bubbles; Chemical effects such as production of hydroxyl radicals in water predominate over physical effects. It is noteworthy that, when the frequency increases, the depth of penetration of the ultrasonic wave decreases, decreasing the maximum pressure reached during implosion. It is therefore necessary to increase the sound power to obtain the same effects as at low frequency ${ }^{7,8}$.

\section{2. 2 .2. Dissipated ultrasonic power}

A piezoelectric transducer converts electrical energy into mechanical energy that is thus transmitted to the liquid, which is irradiated ${ }^{9}$. No transducer is $100 \%$ efficient in converting electrical to mechanical power. To determine the power output $\left(P_{\text {out }}\right)$ for a given power input $\left(P_{i n}\right)$, it is necessary to know the transducer efficiency $(h)$, according to Eq. X.2.

[Insert Equation X.2 here]

The mechanical energy is then converted into acoustical energy generating acoustic cavitation if the minimum power required is reached, i.e., at the Blake threshold, which is $0.5 \mathrm{~W} . \mathrm{cm}^{-2}$ at $20 \mathrm{kHz}$ in pure water and at atmospheric pressure ${ }^{7}$. It is relatively easy to measure the electrical energy delivered by a transducer, but this value in no way reflects the acoustic energy absorbed by the medium, which is the only that can be active. Different methods are proposed for the determination of ultrasonic power $\left(P_{\text {acous }}\right)$ such as thermoacoustic sensor ${ }^{10}$, acousto-optic interaction $^{11}$, piezoelectric hydrophone ${ }^{12}$, sonoluminescence, and chemical dosimetry $^{8}$. Nevertheless, one of the most useful methods has been found to be the calorimetric one ${ }^{8,13}$. Therefore, the absorbed acoustic power $\mathrm{P}_{\text {acous }}$ in $\mathrm{W} \cdot \mathrm{mL}^{-1}$, transmitted to the solution can be measured using a conventional thermal probe 
92 method $^{14,15}$. Using this method, all energy delivered to the system is considered as dissipated as heat (Eq. 3).

[Insert Equation X.3 here]

\section{2. 2. 3. Hydrostatic Pressure}

Hydrostatic pressure is a crucial parameter as the conditions within a collapsing cavitation bubble become more extreme as it increases ${ }^{16}$. Thus, it has been shown that, cavitation threshold in ultrapure water increases linearly with the hydrostatic pressure as well as intensity of bubble collapse ${ }^{8}$. Thereby, an optimal hydrostatic pressure is required to increase the efficiency of ultrasound when used in sonochemical processes ${ }^{17}$.

\section{2. 1. 4. Temperature}

When increasing temperature of a liquid, the solubility of gases it contains decreases whereas its vapor pressure increases decreasing the cavitation efficiency ${ }^{18}$. The influence of temperature on cavitation threshold is especially noticeable at elevated hydrostatic pressures and for fluids with large amounts of dissolved gases ${ }^{19}$.

\section{2. 1.5. Nature of the solvent}

Properties such as (1) solvent viscosity, (2) its vapor pressure, and (3) its surface tension can impact ultrasound intensity. For pure solvents the most determining parameter is the vapor pressure, which when high, decreases cavitation effects ${ }^{8}$. Thus, combination of a high surface tension with a low viscosity and a low vapor pressure favors cavitation ${ }^{18}$.

\section{2. 1. 6. Dissolved gas}


114 After few acoustic cycles, transient bubbles violently collapse into smaller tiny bubbles that act as nuclei of new bubbles and create a hot spot with temperatures of up to $10,000 \mathrm{~K}^{19}$. This adiabatic collapse of the bubbles is assumed to allow the calculation of the temperature and the pressure inside the bubbles according the equations $X 4$ and $X 5^{20}$. Whereas Eq. X.6 allows the calculation of pressure inside the bubble at the moment of the collapse.

\section{[Insert Equations X.4, X.5 and X.6 here]}

Thus, according to equations X.4 and X.5, a monatomic gas leads to higher maximum temperatures and pressures and thereby to more violent collapse of the bubble than a polyatomic gas because its $\mathrm{V}$ value is greater. Thermal effects consecutive to the collapse of the bubble mainly depend on gas phase thermal properties such as heat capacity and thermal conduction.

In the presence of gas with high thermal conduction, the temperature reached at the time of implosion is lower than in the presence of a gas with low thermal conduction, decreasing thus $T_{\max }$ In addition, increasing the gas content, i.e. the number of gas nuclei of a liquid, leads to lowering of both the cavitational threshold and the intensity of the shock wave released on the collapse of the bubble.

\section{2. 2. 7. External pressure}

An increase of $P_{h}$ leads to an increase of $P_{m}$, which is the pressure of the medium (Eq. X.6). In equations X.4 and X.5, when $P_{m}$ increases $T_{m}$ and $P_{m}$ increase, leading to an increase in the cavitation threshold and the intensity of cavity collapse.

\section{2. 2. 8. Ultrasonic intensity}

The ultrasonic intensity, $I_{\max }$, can be expressed as Eq. X. $7^{7}$ : 
If considering $\rho$ and $c$ constant in the medium where the sound propagates, then $I_{\max }$ is proportional to the square of the acoustic amplitude $P_{\mathrm{A}}$. Thus, an increase in $\mathrm{P}_{\mathrm{A}}$ leads to an increase of the ultrasonic intensity, which increases the sonochemical effects up to an optimal value. Indeed, an increase of $\mathrm{P}_{\mathrm{A}}$, leads to a decrease of the time available for the collapse of the too large bubble formed that may become thus insufficient.

Likewise, during their propagation through a medium, the intensity of ultrasound waves decreases when their distance from the emitting source increases (Eq. X.8). Indeed, sound is attenuated in a liquid medium due to reflection, refraction, diffraction and/or scattering of the waves, or the conversion of some of their mechanical energy into heat.

In addition, if too many bubbles are produced at the transducer/liquid interface, the ultrasonic energy entering the system is attenuated, decreasing the efficiency by decoupling of the system.

\section{2. 3. Mode of irradiation and sonoreactors}

\section{2. 3. 1. Modes of irradiation}

Two physic phenomena are widely used to generate ultrasonic waves from ultrasonic devices: magnetostriction and piezoelectricity. Phenomenon of magnetostriction takes place when ferromagnetic materials transform an oscillating magnetic field into mechanical vibration ${ }^{7}$. Piezoelectric effect is the ability of certain crystalline materials when subjected to an electric field to convert electrical energy 
into mechanical energy. The strain is then proportional to the applied field and the mechanical vibrations may lead to ultrasonic sound ${ }^{21}$. This phenomenon is the most widely used in ultrasound devices.

The main materials used are barium titanate $\left(\mathrm{BaTiO}_{3}\right)$, synthetic cristals of lithium nobiate $\left(\mathrm{LiNbO}_{4}\right)$ and lead zirconate titanate $\left(\mathrm{PbTiZrO}_{3}\right)$. These materials are separated from the reactor by metal or glass and ultrasound waves irradiate directly or indirectly the reaction medium (see Figure X.2). An immerged titanium probe is often used as a waveguide.

[Insert Figure X.2 here]

\section{2. 3. 2. Equipment}

Three major sonoreactors are used at lab: ultrasonic bath, cup horn and probe (Figures X.2, and X.3) $22,23,24$.

[Insert Figure X.3 here]

Ultrasonic bath, which represents the most common source of ultrasound in laboratories, is widely used for its low cost. With a frequency of 20 to $60 \mathrm{kHz}$ and acoustic intensities of 1 to $5 \mathrm{~W} . \mathrm{cm}^{-2}$, this device is not well adapted to organic reactions due to the non-homogeneous dissipation of ultrasound energy and the associated lack of reproducibility of the experiments ${ }^{25}$.

Cup-horn reactors present high intensity, which is generally 50 times more intense than in US baths, and directly irradiate the liquid medium ${ }^{26}$. Their geometry allows a good distribution of the ultrasonic field. This device is able to produce low and high frequencies depending of the piezoelectric ceramic chosen and positioned at the bottom of the reactor for upward irradiation. 
Ultrasonic probes provide intense ultrasonic energy, which is concentrated at

their tip and approximately 100 times higher compared to ultrasonic baths; they allow a direct irradiation of the medium.

In both cases, the use of a jacketed reactors is recommended to control the temperature of the medium during the study of organic reactions at lab ${ }^{6}$.

In addition, a study of the shape of the reactor may be relevant to avoid dead zones or to optimize certain physical, thermal and / or chemical effects. Thus, the tubular reactors, providing radial irradiations, make it possible to focus the highintensity ultrasonic field in the heart of the tube and to develop continuous processes. $^{27}$.

\section{2. 3. 3. Characterization of the ultrasonic parameters}

A rigorous characterization of sonochemical parameters is crucial to facilitate the comparison between each study reported in the literature and to understand the involved mechanisms ${ }^{6}$. Indeed, the used frequency, electric and acoustic powers, ultrasonic intensity, radical production, shape and geometry of the reactor and other experimental conditions (temperature, pressure, gas atmosphere, nature and volume of solvent, etc.) have to be rigorously reported in the experimental part of scientific publications.

The frequency, inherent to the equipment used, is the first parameter to report to identify which range is used between low frequency $(20-80 \mathrm{kHz})$ and high frequency $(200-2,000 \mathrm{kHz})$. The electric power also called "nominal electric power" or "electric power input" is the energy delivered by the device, measured by a wattmeter (in W). The absorbed acoustical power ( $P_{\text {acous, }}$ expressed in $\mathrm{W}$ or $\mathrm{W} \cdot \mathrm{mL}^{-1}$ ) can be estimated through a calorimetric method using Equation X.3. Acoustic 
intensity (lacous) is defined as the acoustic power per unit area of the probe (Equation X.9, W. $\left.\mathrm{cm}^{-2}\right)$.

[Insert Equation X.9 here]

As previously mentioned, the extreme conditions during the collapse of bubbles lead to radical production. For example, the sonolysis of water (Scheme X.1) and the recombination of radical species into hydrogen peroxide (Scheme X.2) are observed under ultrasonic conditions.

[Insert Schemes X.1 and X.2 here]

The radical species production can be experimentally estimated or measured by dosimetry methods ${ }^{28,29}$, Electron Paramagnetic Resonance (EPR), ${ }^{30}$ spin-trapping and/or sonochemiluminescence experiments ${ }^{31,32,33}$. Chemical dosimetry is the most convenient and employed method. For example, KI dosimetry involves the oxidation of iodide ions into iodine by hydroxyl radicals formed under ultrasound, through the reaction shown in Scheme X.3. The concentration of $\mathrm{I}_{3}^{-}$can be easily measured using UV-Visible spectrophotometry at a wavelength of $355 \mathrm{~nm}\left(\varepsilon_{\lambda}=26,303 \mathrm{~L}\right.$. mol ${ }^{-}$ ${ }^{1} . \mathrm{cm}^{-1}$ ) to deduce the concentration of $\mathrm{HO}^{\bullet}$ radicals.

\section{[Insert Scheme X.3 here]}

Other methods are used such as Fricke dosimetry (oxidation of $\mathrm{Fe}^{2+}$ to $\mathrm{Fe}^{3+}$ ), terephthalate dosimetry (use of terephthalic acid in alkaline solution) or nitrite and nitrate dosimetry ${ }^{6}$. The Sonochemical Efficiency (SE) can be defined combining electric/acoustic power and rate of radicals formation determined by dosimetry (Equation X10). It constitutes an efficient assessment method to compare different ultrasonic conditions/reactors. 
In EPR spin-trapping experiments diamagnetic nitroso or nitrone compounds are used as spin trap for the conversion short-lived radicals into relatively longer lived nitroxyl radicals that are observable by EPR spectroscopy ${ }^{30,34}$. This accurate characterization method is limited by the non-availability of the equipment at lab.

The sonoluminescence is the emission of photons during the collapse of bubbles. The activity of radical or excited species formed in the gas phase of the bubbles during this collapse can be explored using specific detectors by observing the UV-Visible spectra of the sonoluminescence ${ }^{35,36}$. The use of luminol solution (3aminophtalhydrazide) oxidized by $\mathrm{HO}^{\circ}$ radical formed in water under sonochemical activation leads to the formation of 3-aminophthalic acid with electrons in an excited state. The visible blue light emission $(\lambda=430 \mathrm{~nm})$ due to the de-energization of these electrons allows the mapping of effective zones in a sonoreactor, through this chemiluminescence method ${ }^{36}$.

\section{3. Organic sonochemistry: beneficial effects and new reactivities}

\section{3. 1. Green organic sonochemistry}

The effects of ultrasound during organic reactions have led to serious improvements in terms of reactivity and performance, often under mild conditions, in accordance with a green chemistry approach ${ }^{37}$. Sonochemical reactions in water or biphasic aqueous systems constitute a great potential for further developments.

Sonocatalysis often allows using environmentally friendly conditions, and to decrease reaction times and reach higher yields. In addition, cavitation phenomenon leads to physical and chemical effects, which consequences are interesting for catalyst surface cleaning and free reactive radicals production ${ }^{38,39}$. In the most 
general cases, the combination of ultrasound activation and a catalyst can lead to synergistic effects, mainly observed through the physical effects of US on solid catalysts. Indeed, the physical effects generated by cavitation bubble collapse leads to improved mass transfer that contributes to permanent cleaning of the catalyst's surface and increases the probability for compounds to meet and react. Shear forces induced by shock waves and microstreaming provoke the de-agglomeration of catalyst, the reduction of particle size, which implies an increase in surface area ${ }^{40}$. For these reason, poisoning of catalyst can be avoided, the active surface of catalyst is increased, which leads to higher reaction rates, improved yields and lower chemicals consumption; this makes sonocatalysis an effective tool for green chemistry $^{41,42,43,44,45}$. As an example, the S-alkylation of hetaryl thiols was performed at room temperature six times faster and led to increased yield under US $(74 \%$ in 30 min) compared to silent conditions $(66 \% \text { in } 3 \mathrm{~h})^{42}$. Another synergistic aspect relies in the microbubbles present in the crevices of solid catalysts. They constitute nuclei for cavitational bubble, increasing the number of collapsing events ${ }^{46}$. When bubble collapse near a solid surface such as a solid catalyst particle or vessel, the inrush of liquid generates an "asymmetrical collapse" disaggregation of the catalyst but also the fragmentation of the bubble in smaller bubbles that constitute new nuclei for more cavitation bubbles and collapsing events ${ }^{47,48}$.

In the case of semiconductor catalyst such as $\mathrm{TiO}_{2}$, electron/hole pairs can be generated at its surface through the absorption of energy: (UV-)light in the case of sonoluminescence from cavitation bubbles, or by heat from the extreme temperature resulting from the collapse of bubbles ${ }^{41,49}$. These charges (electron and hole) operate in redox reactions with the solvent to form radical species or directly with organic compounds of the medium. In sonicated reaction medium, the generation of 
280 radical species will favor reaction mechanism implying electron transfer rather than

281 ionic path ${ }^{23}$. For example, the heterocyclization of 1,2-propanediol with fullerene to

282 form fullerene-fused dioxane adduct was enabled by the improved mixing of the nonmiscible liquid phases, provided by US and also possibly by secondary radical reaction in the reaction bulk that would react with the 660 fullerene structure ${ }^{50}$. A change in mechanism and selectivity was describe by Ando where benzyl bromide reacted with potassium cyanide and alumina in toluene with a Friedel-Crafts mechanism to afford 0 - and $p$-benzyltoluene when stirred mechanically at $50{ }^{\circ} \mathrm{C}$. Under $45 \mathrm{kHz}$ US irradiation, the mechanism switched to a nucleophilic substitution and lead to the production of benzyl cyanide in $71 \%$ yield ${ }^{51}$. Bubbles generated by acoustic waves in the reaction medium are influenced by the presence of solid catalyst particles and vice versa, justifying the beneficial effect of their combination, called synergy.

Another meaning of sonocatalysis is the activation of a reaction by US, through physical and chemical effects generated by cavitation activity ${ }^{23}$. In that case, the origin for the effects observed are separated into two categories: true and false sonocatalysis, "true" being linked to chemical effects such as the generation of radicals, and false referring to mechanical effects of US ${ }^{52}$.

\section{3. 2. Cases studies in organic sonochemistry}

\section{3. 2. 1. Examples of oxidation reactions}

Many oxidation reactions such as aldehyde ${ }^{53}$ or alcohol ${ }^{54}$ oxidations have been studied under ultrasonic irradiation because their mechanisms pass through a radical route. 
The aerobic oxidation of D-glucose into D-glucuronic acid was investigated

under high frequency ultrasound $\left(550 \mathrm{kHz}, \mathrm{P}_{\text {acous }}=0.36 \mathrm{~W} \cdot \mathrm{mL}^{-1}\right)$ (see Scheme X.4 $)^{55}$. In absence of oxygen, D-glucose was oxidized into D-gluconic acid as main product $(40 \%$ yield), while D-glucose under oxygen bubbling was oxidized into Dglucuronic acid with excellent yield and selectivity (94\% and 98\%, respectively). The presence of oxygen bubbling into reaction media allows to increase production rate of radicals $\mathrm{HO}^{\circ}$. In this case, mechanisms involving $\mathrm{HO}^{\circ}$ and $\mathrm{HOO}^{\circ}$ were suspected.

\section{[Insert Scheme X.4 here]}

In a very recent study, the $\mathrm{H}_{2} \mathrm{O}_{2}$-mediated epoxidation of cis-cyclooctene performed under high frequency ultrasonic irradiation $\left(800 \mathrm{kHz}, \mathrm{P}_{\text {acous }}=0.58 \mathrm{~W} \cdot \mathrm{mL}^{-1}\right)$ led to improve results compared to silent conditions and revealed important mechanistic insights of the studied reaction (see Scheme X.5) ${ }^{56}$. Indeed, while a maximum yield of $89 \%$ and a selectivity of $91 \%$ were observed under silent conditions, better results were obtained (96\% yield and $98 \%$ selectivity) in 30 min under high frequency ultrasound induced by a good thermoregulation and mild mixing brought by the sonoreactor. In addition, the non-radical nature of ciscyclooctene epoxidation mechanism has been demonstrated.

\section{[Insert Scheme X.5 here]}

At last, the oxidative cleavage of double bond of olefins has been studied under ultrasonic irradiation (see Scheme X.6. ${ }^{56}$. Simultaneous use of ultrasound and Aliquat $336^{\circledR}$ as phase transfer catalyst was essential to perform the oxidative cleavage of olefins without organic solvent and in less than $1 \mathrm{~h}$. At room temperature and thanks to $20 \mathrm{kHz}$ ultrasound probe, mono- and diacids derived from linear and cyclic olefins are obtained with good to excellent yields, from 65 to $96 \%$. 


\section{3. 2. 2. Examples of reduction reactions}

329

330

331

Amines are important molecules that are involved in the synthesis of amino acids ${ }^{57}$ or vitamins ${ }^{58}$ and many of them exhibit interesting biological activities. Therefore, they are used as antihistaminics, analgesics, antiglycemics ${ }^{59}$, anesthesic ${ }^{60}$, decongestant $^{61}$, pyschostimulant ${ }^{62}$ or antidepressant ${ }^{63}$. Classical preparation of amine derivatives relies on the reduction of nitro precursors using different methods. Among them hydrogenation catalyzed by metals, is still the most used in the petrochemical and pharmaceutical industries ${ }^{64}$. However, these methods are often associated with a lack of chemoselectivity in the presence of other reducible functions. In addition, the reaction is exothermic, and involves pressurized hydrogen and flammable solvents, requiring thus particular precautions in terms of safety ${ }^{65}$. The research of more selective, safe and environmentally friendly method of reduction is thus an important issue. It is well known that low frequency ultrasonic irradiation $(20 \mathrm{kHz}<\mathrm{f}<80 \mathrm{kHz}$ ) may enhance catalyst activity and chemical reactions' kinetic and selectivity ${ }^{66}$. Thus, in 2000, Basu et al. described the reduction of nitroaromatic compounds to the corresponding amines using a $\mathrm{Sm} / \mathrm{NH}_{4} \mathrm{Cl}$ reducing agent under 10 to 25 min of ultrasonic irradiation (see Table X.1) ${ }^{67}$.

[Insert Table X.1 here]

In Table X.1, $86 \%$ of 6-aminochrysene were obtained from 6-nitrochrysene (Table X.1, compound 2) after $10 \mathrm{~min}$ of sonication using a $\mathrm{Sm} / \mathrm{NH}_{4} \mathrm{Cl}$ reducing agent in methanol, whereas no reaction was observed under silent conditions. 2nitro-9H-fluorene (Table X.1, compound 3) was reduced to 2-aminofluorene in 10 min under ultrasonic irradiation whereas no transformation was observed when the 
reducing agent was changed for $I n / \mathrm{NH}_{4} \mathrm{Cl}$ or for Fe powder $/ \mathrm{NH}_{4} \mathrm{Cl}$. Under silent conditions, $10 \mathrm{~h}$ under methanol reflux were required to reduce 2-nitro-9H-fluorene (Table X.1, compound 3) to the corresponding amine. In addition, the reaction was shown to be selective towards sensitive functional groups such as bromo (Table X.1, compound 4), cyano (Table X.1, compound 5), ester (Table X.1, compound 6), unsaturated bonds (Table X.1, compound 7) and heterocycles (Table X.1, compound 8). Unfortunately, ultrasonic frequency and power are not given, making it impossible to reproduce the experiments under identical conditions.

Hypophosphorous acid and its salts are mild, cheap and powerful reducing agents commonly used for electrochemical applications ${ }^{68}$. Furthermore, sodium hypophosphite seems has been registered as a non-hazardous substance for Man and for the Environment in 2010 by $\mathrm{REACH}^{69}$. In that context, with sodium hypophosphite/hypophosphorous acid as reducing system and $\mathrm{Pd} / \mathrm{C}$ as catalyst, Letort et al. studied the reduction of nitro compounds to the corresponding amines in $\mathrm{H}_{2} \mathrm{O} / 2-\mathrm{MeTHF}$ at $60{ }^{\circ} \mathrm{C}^{70}$. The authors studied the reaction in terms of solvent effect, $\mathrm{NaH}_{2} \mathrm{PO}_{2} / \mathrm{H}_{3} \mathrm{PO}_{2}$ ratio, temperature, $\mathrm{Pd} / \mathrm{C}$ catalyst loading, with 2-nitroethylbenzene as model substrate. The methodology scope was then extended to other aliphatic nitro compounds (see Table X.2) and the results obtained were compared to those obtained under ultrasonic irradiation.

[Insert Table X.2 here]

Under the same conditions and even with $1.25 \mathrm{~mol} \%$ of $\mathrm{Pd} / \mathrm{C}$ the reduction of $\beta$-nitrostyrene did not lead to the corresponding unsaturated amines nor to the saturated one, but to a mixture of $44 \%$ of $(Z)$ - and $38 \%$ of $(E)$-2-phenylacetaldehyde oximes $^{70}$. The reaction was found to be faster under ultrasonic irradiation and especially in the mixture $\mathrm{H}_{2} \mathrm{O} / \mathrm{Me}-\mathrm{THF}(2: 1)$. Thus, a maximum yield of $92 \%$ of 2- 
phenylethan-1-amine was obtained at $60{ }^{\circ} \mathrm{C}$ in only $15 \mathrm{~min}$ under ultrasonic

377 irradiation compared to 90 min required under silent conditions. In water as solvent 90 min were required to obtain $35 \%$ of amine under silent conditions whereas almost the double was obtained under ultrasonic irradiation. In water and under ultrasonic irradiation, a maximal yield of $90 \%$ was obtained in 15 min when the temperature was increased to $70{ }^{\circ} \mathrm{C}$. The use of a vibromixer allowed the authors to attribute the effects of ultrasound to the formation of an intense micro-emulsion and classified the reaction as a type II reaction termed as "false sonochemistry".

\section{3. 2. 3. Examples of fused heterocycles}

$N$-containing heterocycles are essential building blocks for the synthesis of many biologically active compounds ${ }^{71,72}$. In that context, Nongrum et al. described the synthesis of fused benzo $N, N$-containing heterocycles under ultrasonic activation and using 1-désoxy-1-(méthylamino)-D-glucitol, i.e., meglumine, as organocatalyst ${ }^{73}$. The authors used meglumine, an environmental water-soluble amino sugar, to catalyze the reaction in association with ultrasound as alternative activation technic with mixture ethanol-water as solvent. The reaction was optimized in terms of catalyst loading, solvent and activation technique with 1,2-phenyldiamine, dimedone and tolualdehyde as substrates. The scope of the reaction was then extended to aromatic aldehydes bearing electron-withdrawing and -donating substituents, leading to excellent yields under 20 to $30 \mathrm{~min}$ of ultrasonic irradiation (see Table X.3 $)^{73}$. Two hypotheses have been formulated to explain the role of meglumine, which is suggested to act via electrophilic or nucleophilic activation of dimedone. Under the same conditions, 85 to $90 \%$ of quinoxaline derivatives were synthesized under 25 to 35 min of ultrasonic irradiation at $50{ }^{\circ} \mathrm{C}$.

[Insert Table X.1 here] 
Using an acidic ionic liquid, the 1,4-diazabicyclo[2,2,2]octanium diacetate, i.e.,

402

403

404

405

406

407

408

409

410

411

412

DABCO diacetate, Fekri et al. synthesized 93 to $97 \%$ of various benzodiazepines

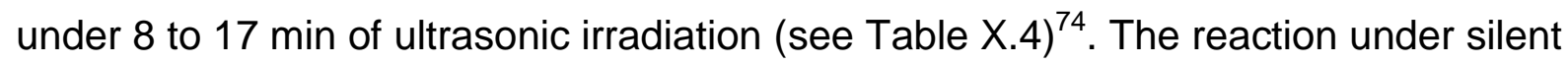
conditions and at $90{ }^{\circ} \mathrm{C}$ afforded the products in higher reaction times and lower yields. A mechanism involving the ionic liquid for increasing the electrophilicity of the carbonyl substrates is then proposed.

[Insert Table X.4 here]

\section{3. 2. 4. Examples of organometallic reactions}

Sonochemistry is a green alternative method to classical methods to promote the synthesis of organic compounds, offering then versatile and easy pathways for a wide variety of transformations such as Reformatsky, Barbier-Grignard or Michael reactions $s^{75}$.

The Reformatsky reaction, which converts aldehydes and ketones to $\beta$ hydroxyesters, is one of the first organometallic reactions with problems of metal activation under silent conditions that was studied under ultrasound. In 1982, it was shown that the addition of ultrasound resulted in better reaction yields in less time compared to silent conditions ${ }^{76}$. In fact, for a reaction time of $12 \mathrm{~h}$, the yield was $58 \%$ while with ultrasound (ultrasonic bath, frequency not indicated), it rose to $98 \%$ in only $30 \mathrm{~min}$. Several studies were carried out in order to understand the role of ultrasound in this reaction ${ }^{77,78,79}$. Authors reported successful syntheses with quantitative yields of $\beta$-hydroxyester by reacting phenylketons, $\alpha$-bromoesters, zinc dust and a catalytic amount of iodine under high intensity ultrasonic irradiation ${ }^{80}$. But, the role of iodine was not fully understood. Reformatsky reactions could then be carried out using nontraditional electrophiles such as glyoxylic oximes ${ }^{81}$. 
In the Barbier-Grignard type reaction, the organometallic reagent, which

426

427

428 intervenes, serves as a nucleophile for the formation of a carbon-carbon bond ${ }^{82}$. The zinc-mediated Barbier reaction was carried out in a biphasic $\mathrm{CO}_{2} / \mathrm{H}_{2} \mathrm{O}$ system allowing a preferential partitioning of the desired homoallyl alcoholic product ${ }^{83}$. Here, pulsed ultrasound is efficient for dispersing and mixing the two phases creating thus an emulsion while ensuring the kinetic control.

The Michael reaction is often a first choice organic transformation because there are a large number of Michael acceptors and nucleophiles. Indeed, almost all activated alkenes such as $\alpha, \beta$-unsaturated ketones can act as acceptors. It is also possible in a single step to form stereogenic centers ${ }^{84}$. Numerous studies have demonstrated the need for basic or acidic catalysts in the reaction of addition of nucleophiles 1,4-conjugated to unsaturated carbonyl compounds ${ }^{85}$. However, these conditions gave way to side reactions. The effects of ultrasound then allowed a more efficient approach when adding the conjugate ${ }^{86}$. In a recent study, researchers studied the chemical behavior of 3-diethyl phosphonocoumarin in an ultrasoundassisted Michael-type reaction ${ }^{87}$. Several organometallic compounds have been selected to react with this coumarin as hard nucleophiles isomers (see Scheme X.7). This approach allowed the use of a simpler experimental set and milder reaction conditions. Excellent reproducibility of yield is reported as well as high diastereoselective activity of isolated trans isomers ${ }^{87}$.

\section{[Insert Scheme X.7 here]}

\section{3. 3. Scale-up and industrial applications}

In organic sonochemistry, a series of macroscopic characteristics, namely the use of less toxic/hazardous chemicals, eco-friendly solvents, alternative or renewable raw 
materials, the development of reaction conditions to increase the selectivity of the product or even the minimization of consumption energy during transformations reinforce the aspect sought in green chemistry and encourage the use of ultrasound on a larger scale 6,88

As presented previously, lab scale experiments in organic chemistry are very promising to create green processes in industry ${ }^{23,89}$. However, to our knowledge, no industrial scale sonoreactor was successfully employed for organic synthesis. Large scale transfers require adjustments concerning various parameters and a scale-up methodology is lacking ${ }^{90}$.

The efficiency of sonochemical reactions relies on hydrodynamics, mixing and mass transfer. Two types of parameters need to be studied when scaling up a labscale experiment to industrial purpose: (i) the operating parameters such as the frequency, the intensity and the initial radius of bubble nuclei and (ii) the geometric parameters such as the size and location of transducers, the shape and dimensions of the reactor. Both types of parameters will impact the mixing and hydrodynamic characteristics that define the cavitation field. The main problems in intensifying organic reactions relate to the fact that the yield and selectivity of the chemical reaction are non-linearly based on operating parameters and geometry; a proportional amplification of the dimension of the reactor is therefore not sufficient to reproduce the same experimental conditions. Fine predictions of many parameters such as pressure, temperature, and cavitational activity distribution are required to achieve chemical reaction effectiveness. Another problem frequently encountered is that cavitation events happen mainly near the irradiating surfaces. The adjustment of lab-scale reaction at large-scale requires higher energy input to counterbalance energy dissipation in wider bulk media leading to fast erosion of the sonication 
474 surfaces ${ }^{90}$. Large-scale reactors contain larger volumes, which bring the issue of US

475 attenuation, due to reflection, refraction or absorption of the incident sound wave and leads to spatial variation of the cavitational activity, creating active and passive zones in the reactor. This needs to be avoided to perform chemical reactions at an 478 industrial scale ${ }^{91}$.

To anticipate the problematics related to large scale sonoreactors, experimental measurements and theoretical techniques exist to predict the cavitational activity distribution. The mapping of a sonoreactor can contribute to upscale chemical reactions. It consists in the characterization of the cavitational phenomena and implies the quantification of primary effects (generated at the same time as the bubble collapse) and secondary effects (generated after bubble collapse). Parameters like pressure can be quantified using a hydrophone while changes in local temperature can be measured with a thermocouple ${ }^{92}$ and gives information on the local cavitation activity and is used to quantify the efficacy of the sonochemical reactor in term of energy transfer. The quantification of secondary parameters as radical production can be assessed through dosimetry. Chemical reaction implying hydroxyl radicals enable an indirect measure of their concentration and they can have large measurement errors ${ }^{93}$. The modelling of cavitational parameters such as pressure, temperature but also the bubble radius and the chemical species formed can be predicted through modelling ${ }^{94,95}$. Large scale reactors imply specific studies as for example, the pressure of the reaction medium is a function of depth and impacts the conditions of cavitation bubble collapse like pressure and temperature, and thus the physical and chemical effects on chemical reactions ${ }^{96,97}$. Concerning

497 sonochemical efficiency assessment, most studies are conducted on single bubble 
498 model $^{98,99}$ which does not take account of neighbor bubbles behavior mutually

499 influencing the other bubble behavior ${ }^{100,101}$.

$500 \quad$ The scaling-up of organic reaction under US would enable great energy and 501 chemical savings. The main difficulty to design large scale reactors is to ensure a 502 uniform distribution of cavitational activity. Empirical methods and theoretical 503 modelling can be used to work on this aspect and contribute to the industrialization 504 of US for organic synthesis.

505 4. Conclusions: from the challenges to new perspectives of organic 506 sonochemistry

507 Since the evaluation of the eco-compatibility of a process cannot be done 508 subjectively, there are a certain number of calculations of green chemistry such as 509 the economy of atoms, the mass reaction efficiency, the intensity mass, effective 510 mass yield, carbon efficiency, the E-factor, the Eco-Scale or other green

511 metrics $^{102,103}$. These tools are still little exploited by sonochemists and the

512 systematization of their use is recommended. The systematic comparison between 513 silent and ultrasonic conditions are necessary, under the same conditions, to 514 highlight the improvements brought by ultrasound in terms of efficiency and ecocompatibility. For example, the energy consumption should be also measured. At lab scale, in situ data monitoring can be collected to extrapolated the ultrasound conditions at a higher scale $\mathrm{e}^{104}$.

In terms of equipment, the combined efforts of chemists, physicists and equipment manufacturers will be needed for the chemical process industry to exploit cavitation as a more viable option for chemical transformations. 
As a perspective, the combination of ultrasound with other physical and

522

chemical methods of activation seems promising. For example, the use of subcritical $\mathrm{H}_{2} \mathrm{O}$ (hydrothermal, $200{ }^{\circ} \mathrm{C}$ ) under ultrasonic irradiation $(20 \mathrm{kHz})$ shows stable cavitation with nonlinear bubble oscillations. On the other hand, formation of hydrogen peroxide $\mathrm{H}_{2} \mathrm{O}_{2}$ thermally instable is not observed ${ }^{105}$. Sonohydrothermal synthesis is described for the synthesis of inorganic nanomaterial ${ }^{106}$, but not yet for organic synthesis. Indeed, the combination of ultrasound with supercritical $\mathrm{CO}_{2}$ has not been used in organic chemistry due to high pressure and lack of phase boundaries observed in the supercritical state ${ }^{107}$. Hence, for biomass valorization, positive influence of ultrasound in supercritical $\mathrm{CO}_{2}$ is observed thanks to mechanical effects (micro- and macro-mixing, cell damage) that could be useful in organic synthesis $^{108}$.

In organic synthesis, the use of US and microwaves in tandem or simultaneously at laboratory scale improves selectivity, yield and lowers reaction times ${ }^{109,110}$, satisfying some principles of green chemistry criteria. The synergistic effects observed are commonly attributed to the improved heat transfer provided by microwaves and the intense mass transfer resulting from US. Each irradiation technology compensates the weakness of the other, which justifies the added value of their simultaneous use, especially in heterogeneous systems ${ }^{110,111}$.

Despite the many advantages of microreactors, one major drawback is the potential clogging of their channels ${ }^{112}$ In that context, the synergy of ultrasound and microfluidics has shown its potential. Thus, Sedelmeier et al. ${ }^{113}$ used an ultrasonic bath to avoid the fouling of the microreactor due to the deposition of manganese dioxide used for the oxidation of nitralkanes into their corresponding carboxylic acids. Recently, Delacour et al. ${ }^{114}$ developed a scaled-up ultrasonic reactor to prevent Royal Society of Chemistry - Book Chapter X 
546 clogging in particle forming reactions. The millireactor was used for the synthesis of 547 barium sulphate. Results showed that only $0.48 \mathrm{~W} \cdot \mathrm{mL}^{-1}$ was required to prevent 548 channel clogging and to increase the productivity by two orders of magnitude 549 compared to a microreactor.

Sonophotocatalysis, which is the combination of sonochemistry and photocatalysis, has mainly been used as an advanced oxidation process for the removal of pollutants. Its recent use in organic synthesis showed promising results as a synergistic effect of both activation methods afforded an alkyl carbon chain elongation from $3 \mathrm{C}$ (malonic acid) to $4 \mathrm{C}$ (succinic acid). The carbon chain elongation was observed only when US and UV irradiations were combined ${ }^{115}$.

At last, the physicochemical properties of some ionic liquids (ILs), such as their large thermal stability, their wide liquid domain or even their low vapor pressure make them very interesting reaction media for green chemistry. The combination of ultrasound with the appropriate ionic liquid is a powerful tool for performing various organic transformations ${ }^{116}$. The rate of reactions, selectivity and yields are often improved. Their combination with US then makes it possible to obtain the desired products without using inorganic of organic catalysts, even at room temperature ${ }^{117}$. The possible degradation phenomena (few ppm) of ILS under US conditions have nevertheless to be considered ${ }^{118}$. Deep eutectic solvents are also very promising as a green alternative in synergy with ultrasound in organic syntheses ${ }^{119}$. to assess to efficiency of the coupling is to calculate its synergy. Synergy corresponds to a beneficial effect from a coupling that is positive if the results obtained with the coupling of processes is superior to the sum of the results obtained 
570 with each independent process ${ }^{109}$. Several indicators of synergy can be found in the

571 literature but their definition and the equations that are used should always be

572 detailed to avoid confusion. Among indicators for synergy the following can be found:

573 synergy, synergy effect, synergistic effect, \%synergy or synergy index ${ }^{120,121,122}$. For

574 example, the equation for percentage of synergy (Equation X.11) and the synergy

575 index (Equation X.12) for $\mathrm{n}$ processes leading to chemical reactions with $\mathrm{k}$ as rate 576 constant, are detailed below.

The calculation of the \%synergy and synergy index are recommended when ultrasound are combined in an organic reaction with another physical or chemical activation method in order to prove the advantages of the used combination.

Figure X.1: Schematic representation of bubble formation, growth and collapsing.

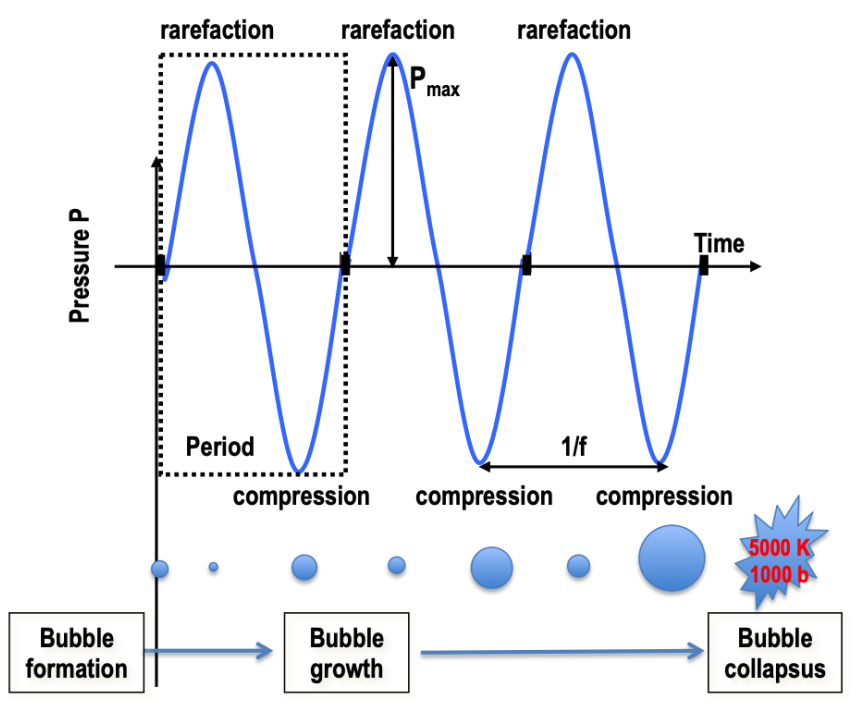


587 Figure X.2: Schematic representation of mode of irradiation depending on the main 588 device used in laboratories (adapted from reference ${ }^{123}$ ).

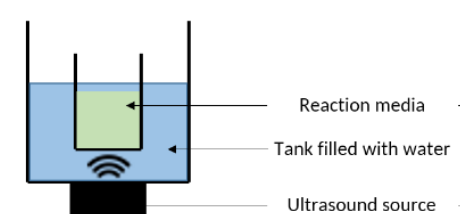

Indirect ultrasonic irradiation (ultrasound bath or cup horn)

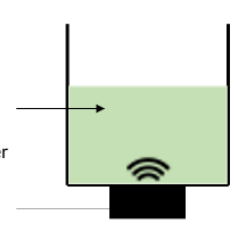

Direct ultrasonic irradiation

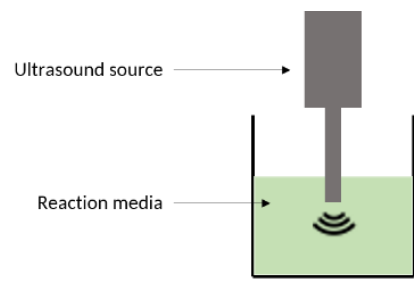

Direct ultrasonic irradiation

591 Figure X.3: Cup horn system (left) and ultrasonic probe (right) at lab.

592

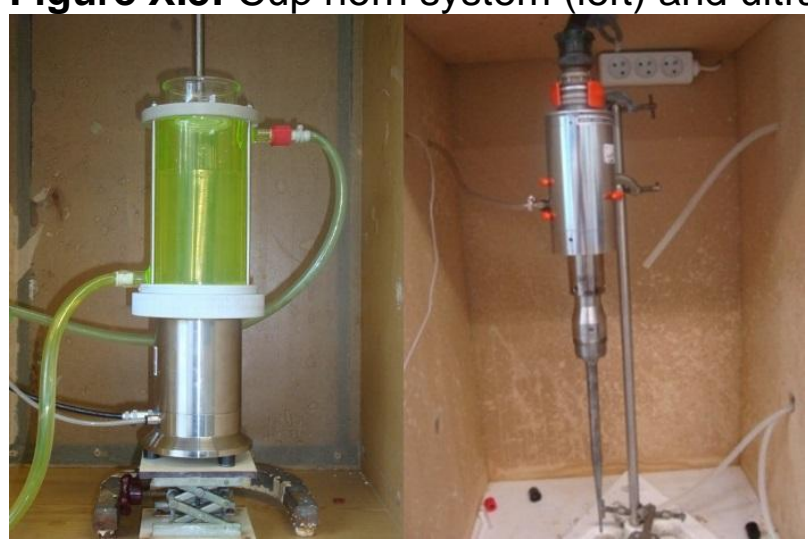

593 Table X.1: Reduction of aromatic nitro compounds by $\mathrm{Sm} / \mathrm{NH}_{4} \mathrm{Cl}$ under ultrasonic 594 irradiation (ultrasonic bath, frequency not indicated, room temperature) ${ }^{67}$.

Time, $\min$

596

597

598 Table X.2: Reduction of aliphatic nitro compounds under silent and ultrasonic conditions (ultrasonic microtip, $\left.20 \mathrm{kHz}, \mathrm{P}_{\text {acous }}=3.94 \mathrm{~W}, 3 \mathrm{~mL}\right)^{70}$. 
$\mathrm{RNO}_{2} \frac{\mathrm{NaH}_{2} \mathrm{PO}_{2} / \mathrm{H}_{3} \mathrm{PO}_{2}(4: 1), \mathrm{Pd} / \mathrm{C} \text {, time }}{\left.\left.\left.\left.\mathrm{H}_{2} \mathrm{O} / 2-\mathrm{MeTHF}(2: 1),\right)\right)\right)\right) \text { or silent, } 60^{\circ} \mathrm{C}} \mathrm{RNH}_{2}$

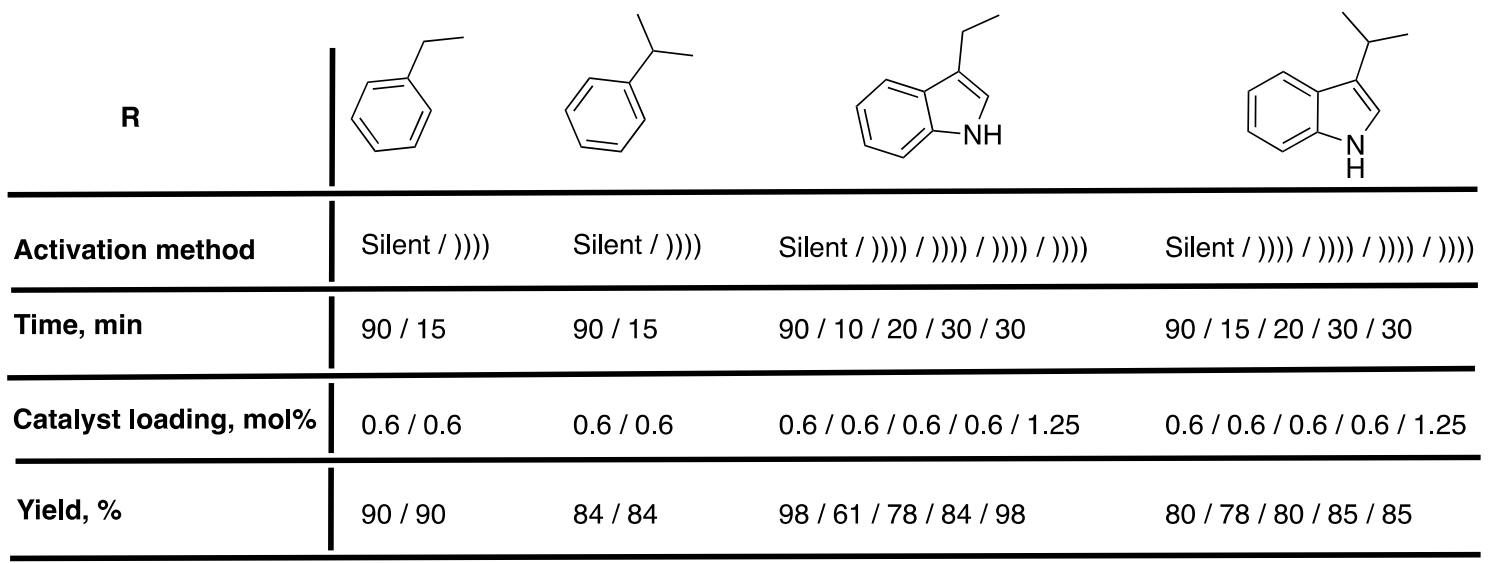

601 Table X.3: Synthesis of $1 H$-dibenzo[b,e][1,4]diazepin-1-one derivatives with 5 mol\%

602 of meglumine as catalyst under silent and ultrasonic conditions (ultrasonic bath) ${ }^{73}$.

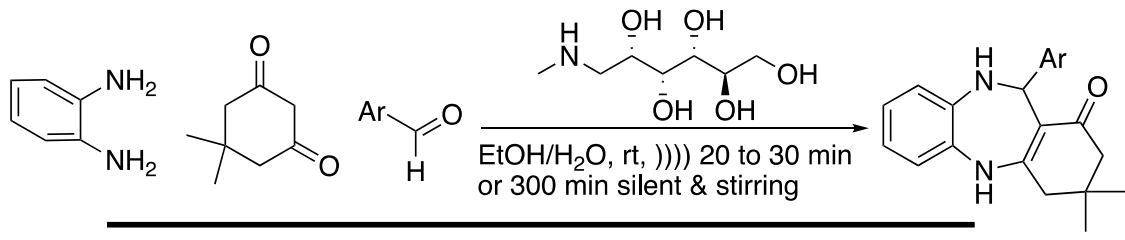

\begin{tabular}{|c|c|c|c|}
\hline \multirow[b]{2}{*}{$\mathrm{Ar}$} & \multicolumn{2}{|c|}{ )))) } & Silent $300 \mathrm{~min}$ \\
\hline & Time, min & Yield, \% & Yield, \% \\
\hline $\mathrm{C}_{6} \mathrm{H}_{5}$ & 20 & 92 & 65 \\
\hline 4- $\mathrm{CH}_{3} \mathrm{C}_{6} \mathrm{H}_{4}$ & 20 & 92 & 68 \\
\hline $4-\mathrm{OCH}_{3} \mathrm{C}_{6} \mathrm{H}_{4}$ & 20 & 88 & 65 \\
\hline 4- $\mathrm{ClC}_{6} \mathrm{H}_{4}$ & 25 & 90 & 60 \\
\hline 4- $\mathrm{BrC}_{6} \mathrm{H}_{4}$ & 25 & 92 & 68 \\
\hline $4-\mathrm{NO}_{2} \mathrm{C}_{6} \mathrm{H}_{4}$ & 30 & 85 & 58 \\
\hline $4-\mathrm{OHC}_{6} \mathrm{H}_{4}$ & 25 & 88 & 60 \\
\hline $4-\mathrm{N}\left(\mathrm{CH}_{3}\right)_{2} \mathrm{C}_{6} \mathrm{H}_{4}$ & 30 & 88 & 64 \\
\hline $2-\mathrm{ClC}_{6} \mathrm{H}_{4}$ & 25 & 82 & 62 \\
\hline 2- $\mathrm{NO}_{2} \mathrm{C}_{6} \mathrm{H}_{4}$ & 30 & 85 & 55 \\
\hline 1-Naphthyl & 25 & 88 & 55 \\
\hline Furfuryl & 30 & 88 & 64 \\
\hline $\mathrm{C}_{6} \mathrm{H}_{5} \mathrm{CH}=\mathrm{CH}$ & 30 & 82 & 60 \\
\hline 4- $(\mathrm{OH})$ and 3- $\left(\mathrm{OCH}_{3}\right) \mathrm{C}_{6} \mathrm{H}_{3}$ & 25 & 90 & 65 \\
\hline $3,4-\left(\mathrm{OCH}_{3}\right) 2 \mathrm{C}_{6} \mathrm{H}_{3}$ & 20 & 90 & 60 \\
\hline
\end{tabular}

603

Table X.4: Synthesis of various benzodiazepines with $0.5 \mathrm{mmol}$ of ionic liquid as

605 catalyst under silent and ultrasonic conditions (ultrasonic bath, $45 \mathrm{kHz}, \mathrm{P}_{\text {in }}=305$ $606 \mathrm{~W})^{74}$ 


\begin{tabular}{|c|c|c|c|c|}
\hline \multirow[b]{2}{*}{$\mathrm{Ar}$} & \multicolumn{2}{|c|}{ ()))) } & \multicolumn{2}{|c|}{ Silent, $90^{\circ} \mathrm{C}$} \\
\hline & Time, $\min$ & Yield, \% & Time, $\min$ & Yield, \% \\
\hline $\mathrm{C}_{6} \mathrm{H}_{5}$ & 10 & 95 & 75 & 82 \\
\hline $4-\mathrm{ClC}_{6} \mathrm{H}_{4}$ & 8 & 97 & 60 & 84 \\
\hline $4-\mathrm{OCH}_{3} \mathrm{C}_{6} \mathrm{H}_{4}$ & 8 & 96 & 60 & 85 \\
\hline $4-\mathrm{NO}_{2} \mathrm{C}_{6} \mathrm{H}_{4}$ & 8 & 98 & 60 & 89 \\
\hline $3-\mathrm{NO}_{2} \mathrm{C}_{6} \mathrm{H}_{4}$ & 10 & 95 & 75 & 88 \\
\hline $3-\mathrm{ClC}_{6} \mathrm{H}_{4}$ & 10 & 96 & 75 & 89 \\
\hline $4-\mathrm{N}\left(\mathrm{CH}_{3}\right)_{2} \mathrm{C}_{6} \mathrm{H}_{4}$ & 12 & 93 & 90 & 85 \\
\hline 2,4-diClC ${ }_{6} \mathrm{H}_{4}$ & 15 & 94 & 90 & 83 \\
\hline & 6 & 95 & 60 & 82 \\
\hline & 17 & 94 & 90 & 81 \\
\hline
\end{tabular}

608

609

610 Scheme X.1:

611

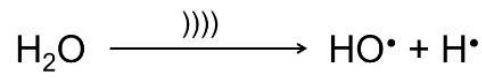

612

613

Scheme X.2:

614

$\mathrm{HO}^{\bullet}+\mathrm{HO}^{\circ}$ $\mathrm{H}_{2} \mathrm{O}_{2}$

616

617

Scheme X.3:

618

619

$2 \mathrm{HO}^{\bullet}+3 \mathrm{I}^{-}$ $2 \mathrm{HO}^{-}+\mathrm{I}_{3}^{-}$

621

\section{Scheme X.4:}

622<smiles>O=C(O)C(O)C(O)C(O)C(O)CO</smiles>
$\left(550 \mathrm{kHz}-0.36 \mathrm{~W} \cdot \mathrm{mL}^{-1}\right)$ $40 \%$<smiles>OCC1OC(O)C(O)C(O)C1O</smiles>

$\left(550 \mathrm{kHz}-0.36\right.$ W. $\left.\mathrm{mL}^{-1}\right)$

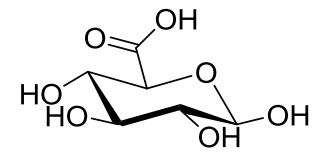

$94 \%$ 


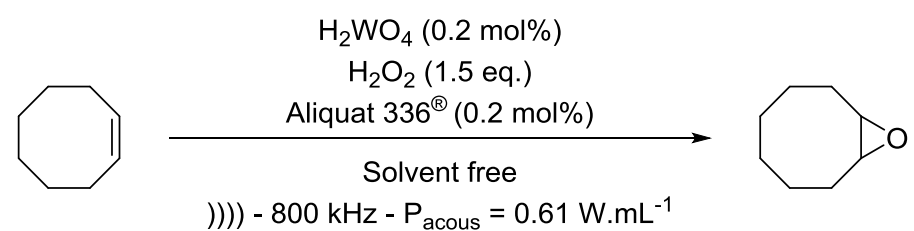

626 Scheme X.6:
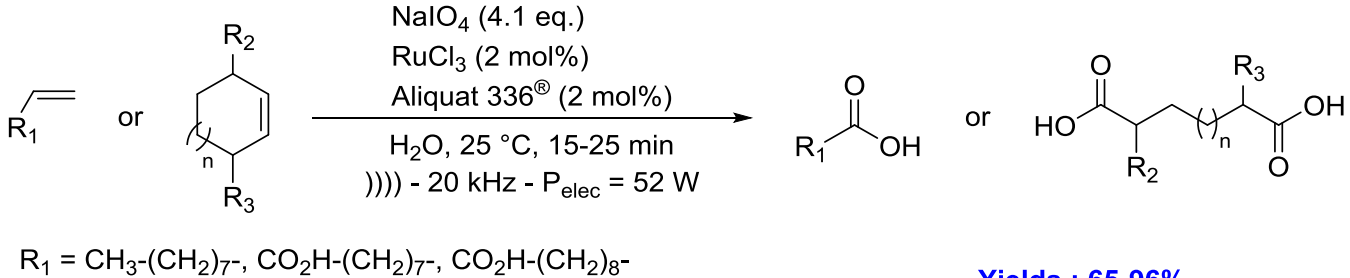$$
\mathrm{R}_{2}, \mathrm{R}_{3}=\mathrm{H}: \mathrm{n}=1-3 \quad \mathrm{R}_{2}=\mathrm{R}_{3}=\mathrm{CH}_{2}: \mathrm{n}=1
$$

Yields : 65-96\%

\section{Scheme X.7:}

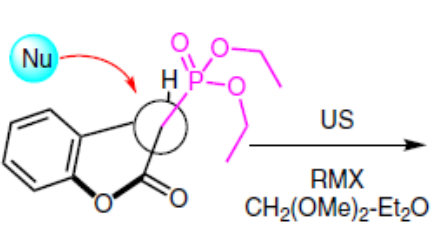

$\mathrm{Nu}=\mathrm{RMX}, \mathrm{E}=\mathrm{H}$

$\mathrm{R}=\mathrm{Et}, n-\mathrm{Pr}, j-\mathrm{Pr}, \mathrm{Bn}, \mathrm{CH}_{2} \mathrm{CO}_{2} \mathrm{Et}$, $\mathrm{CH}_{2} \mathrm{CO}_{2} t-\mathrm{Bu}$

$\mathrm{M}=\mathrm{Mg}, \mathrm{Zn}$

$\mathrm{X}=\mathrm{Cl}, \mathrm{Br}$

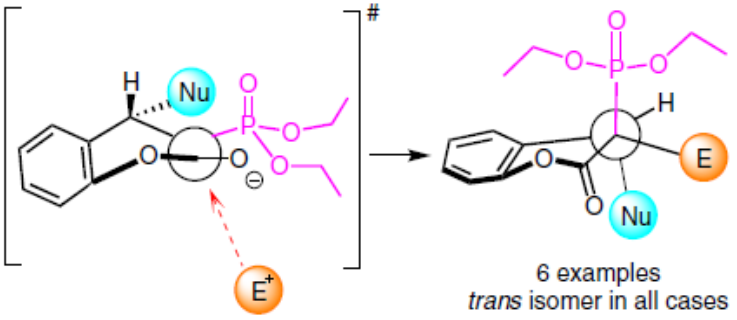

630

\section{Equation X.1.}

$632 \mathrm{f}=\frac{\mathrm{c}}{\lambda}$

633 where $\mathrm{f}$ is the frequency $(\mathrm{Hz}), \mathrm{c}$ is the celerity of sound $\left(\mathrm{m} \cdot \mathrm{s}^{-1}\right)$ and $\lambda$ the wavelength $(\mathrm{m})$.

634

635 Equation X.2.

$636 \mathrm{P}_{\text {out }}=\mathrm{h} \times \mathrm{P}_{\text {in }}$

637

638 Equation X.3.

639

$$
\mathrm{P}_{\text {acous }}=\mathrm{m} \times \mathrm{c}_{\mathrm{p}} \times\left(\frac{\mathrm{dT}}{\mathrm{dt}}\right)_{0}
$$


640 where $P_{\text {acous }}$ is the absorbed acoustical power in $\mathrm{W}$ (or reported to the volume in W.mL $\mathrm{m}^{-1}$ ), $\mathrm{m}$

641 is the mass in $\mathrm{kg}$ of liquid in the sonoreactor, $\mathrm{c}_{\mathrm{p}}$ is the specific heat capacity in $\mathrm{J}_{\mathrm{kg}} \mathrm{kg}^{-1} \cdot \mathrm{K}^{-1}$, $642\left(\frac{\mathrm{dT}}{\mathrm{dt}}\right)_{0}$ is the initial slope of the increase of the temperature of the solution versus time of 643 ultrasonic irradiation.

644

645 Equations X.4., X.5 and X.6

646

$P_{\max }=P\left[\frac{P_{m}(\gamma-1)}{P}\right]^{\frac{\gamma}{\gamma-1}}$

Eq. X.4

647
$T_{\max }=T_{0}\left[P_{m} \frac{(\gamma-1)}{P}\right]$
Eq. X.5

648

649

$P_{m}=P_{a}+P_{h}$

Eq. X.6

650 where $P_{\max }$ and $T_{\max }$ are the maximum pressure and temperature at the collapse, $\mathrm{P}$ is the

651 pressure inside the bubble at its maximum size; it is usually equal to the vapor pressure $P_{v}$,

$652 \mathrm{P}_{\mathrm{m}}$ is the pressure inside the liquid at the moment of the collapse, $T_{0}$ is the ambient

653 temperature, $P_{a}$ is the acoustic pressure applied, $P_{h}$ is the pressure within the fluid. It is 654 usually taken to be ambient or atmospheric pressure. The polytropic factor $\gamma$ is the ratio of 655 the specific heat capacities of the gas or the gas vapor mixture.

656

657 Equation X.7.

$658 \quad I_{\max }=\frac{P_{A}^{2}}{2 \rho c}$

659 where $P_{A}$ is the acoustic amplitude, $\rho$ is the density of the medium, and $c$ is the velocity of 660 the sound in the medium.

662 Equation X.8. 
663

$I_{\max }=I_{0} e^{-2 \alpha d}$

664 where $I_{0}$ is the initial sound intensity, $\alpha$ is the absorption coefficient of the medium and $I_{\max }$ is 665 the ultrasonic intensity at the distance $d$.

666

667 Equation X.9.

668

$$
I_{\text {acous }}=\frac{P_{\text {acous }}}{S_{\text {probe }}}
$$

669 where $\mathrm{I}_{\text {acous }}$ is the acoustic intensity $\left(\mathrm{W} . \mathrm{cm}^{-2}\right), \mathrm{P}_{\text {acous }}$ is the acoustic power $(\mathrm{W})$ and $\mathrm{S}_{\text {probe }}$ is 670 the surface of the irradiating probe.

\section{Equation X.10.}

$$
S E_{\text {elec/acous }}=\frac{n_{\text {ions }}}{E_{\text {elec } / \text { acous }}}
$$

672 where SE is the Sonochemical Efficiency $\left(\mathrm{mol}^{\mathrm{J}^{-1}}\right), \mathrm{n}_{\text {ions }}$ is the number of moles of considered 673 produced under ultrasonic irradiation (mol of $\mathrm{I}_{3}^{-}, \mathrm{NO}_{2}^{-}, \mathrm{NO}_{3}^{-}$depending of the dosimetry 674 method) and $E_{\text {elec/acous }}$ is the electrical or acoustic power (W, electric or acoustic).

\section{Equation X.11.}

676

$\%$ synergy $=100 \frac{k_{\text {combined processes }-\sum_{i=1}^{n} k_{\text {process } i}}}{k_{\text {combined processes }}}$

\section{Equation X.12.}

678

synergy index $=\frac{k_{\text {combined processes }}}{\sum_{i=1}^{n} k_{\text {process } i}}$

\section{ABBREVIATIONS:}

681

c: celerity of sound

682

$\mathrm{C}_{\mathrm{p}}$ : specific heat capacity 
683

DABCO: 1,4-Diazabicyclo[2.2.2]octane

$684 \quad E_{\text {elec/acous: }}$ electrical or acoustic power

685

EPR: Electron Paramagnetic Resonance

686

f: frequency

$687 \mathrm{I}_{0}$ : initial sound intensity

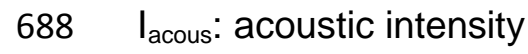

$689 I_{\max }$ : ultrasonic intensity

690 ILs: Ionic Liquids

$691 \mathrm{~m}$ : mass of liquid in the sonoreactor

692 P: pressure inside the bubble at its maximum size

$693 P_{a}$ is the acoustic pressure applied

$694 \mathrm{P}_{\mathrm{A}}$ : acoustic amplitude

$695 \mathrm{P}_{\text {acous }}$ : acoustical power

$696 P_{h}$ : pressure within the fluid

$697 P_{\text {in }}$ : power input

$698 \mathrm{P}_{\mathrm{m}}$ : pressure inside the liquid at the moment of the collapse

$699 P_{\max }$ : maximum pressure at the collapse

$700 \quad P_{\text {out: }}$ power output

$701 \mathrm{P}_{\mathrm{v}}$ : vapor pressure at the moment of the collapse

702 REACH: Registration, Evaluation and Authorisation of Chemicals 
$S_{\text {probe: }}$ surface of the irradiating probe

$705 \mathrm{~T}_{0}$ : ambient temperature

$706 \mathrm{~T}_{\max }$ : temperature at the collapse

707 US: Ultrasound

708 UV: Ultraviolet

$709 \lambda$ : wavelength

$710\left(\frac{\mathrm{dT}}{\mathrm{dt}}\right)_{0}$ : initial slope of the increase of the temperature of the solution versus time of ultrasonic

711 irradiation

$712 \alpha$ : absorption coefficient of the medium

$713 \gamma$ : polytropic factor

$714 \rho$ : density of the medium

\section{REFERENCES:}

1. R. Woods and A. Loomis, Philos. Mag., 1927, 4, 414.

2. T. Richards and A. Loomis, J. Amer. Chem. Soc., 1927, 49, 3086.

3. E. Neppiras, Phys. Rep., 1980, 61, 159.

4. S. K. Bhangu and M. Ashokkumar, Top. Curr. Chem., 2016, 374, 1.

5. T. Lepoint and F. Lepoint-Mullie, Theoretical Bases, In: Synthetic Organic Sonochemistry, Plenum Press, 1998, 1-49. 
6. G. Chatel, Sonochemistry: New opportunities for green chemistry, World Scientific, 2017, $170 \mathrm{p}$.

7. M. Draye, J. Estager and N. Kardos, In: Activation Methods: Sonochemistry and High Pressure, (Eds J.-P. Goddard, M. Malacria and C. Ollivier), Willey, 2019, 26.

8. B. G. Pollet, Electrocatal. 2014, 5, 330.

9. N. Ghasemi, F. Zare, P. Davari, P. Weber, C. Lagton and A. Gosh, Proceedings of the 7th IEEE Conference on Industrial Electronics and Applications (ICIEA), 2012, 647.

10. B. Fay and M. Rinker, Ultrason., 1996, 34, 563.

11. L. He, F. Zhu, Y. Chen, K. Duan, X. Lin, Y. Pan and J. Tao, Rev. Sci. Instrum., 2016, 87, 054903.

12. G. Harris, Ultras. Med. Biol., 1985, 11, 803.

13. T. Uchida, T. Kikuchi, M. Yoshioka, Y. Matsuda and R. Horiuchi, Acoust. Sci. Tech., 2015, 36, 445.

14. N. Navarro, T. Chave, P. Pochon, II. Bisel, and S. Nikitenko, J. Phys. Chem. B, 2011, 115, 2024.

15. I. Margulis and M. Margulis, Acoust. Phys., 2005, 51, 695.

16. C. C. Church, D. F. Gaitan, Y. A Pishchalnikov and T. J. Matula, J. Acoust. Soc. Am., 2011, 129, 2620.

17. H. Delmas, N. Tuan Le, L. Barthe and C. Julcour-Lebigue, Ultrason. Sonochem., $2015,25,51$. 
18. Y. T. Shah, A. B. Pandit and V. S. Moholkar, In: Cavitation Reaction Engineering, The Plenum Chemical Engineering Series, Dan Luss Editor, Springer Science, 1999, 79.

19. J. Rooz, E. V. Rebrov, J. C. Schouten and J. T.F. Keurentjes, Ultrason. Sonochem. 2013, $20,1$.

20. B. E. Noltingk and E. A. Neppiras, Proc. Phys. Soc. B 1950, 63, 674.

21. L. H. Thompson and L. K. Doraiswamy, Ind. Eng. Chem. Res. 1999, 38, 1215.

22. T. J. Mason and J. P. Lorimer, Applied sonochemistry: the uses of power ultrasound in chemistry and processing. 2002.

23. G. Cravotto and P. Cintas, Chem. Soc. Rev., 2006, 35, 180.

24 P. R. Gogate, P. N. Patil, Sonochemical reactors, In: Sonochemistry, Springer, $2017,255$.

25 V. S. Moholkar, S. P. Sable and A. B. Pandit, AIChE J. 2000, 46, 684.

26 J.-P. Bazureau and M. Draye, Ultrasound and Microwaves: Recent Advances in Organic Chemistry, Transworld Research Network, Kerala, 2011, 241.

27 G. Chatel, M. Draye, R. Duwald, C. Piot and P. Fanget, Patent FR 2006171 , 2020.

28 Y. lida, K. Yasui, T. Tuziuti and M. Sivakumar, Microchem. J., 2005, 80, 159.

29 Y. Asakura, M. Maebayashi, T. Matsuoka and S. Koda, Electron. Commun. Japan, 2007, 90, 1.

30 K. Makino, M. M. Mossoba, and P. Riesz, J. Amer. Chem. Soc., 1982,104, 3537. 
31 E. N. Harvey, J. Amer. Chem. Soc.,1939, 61, 2392.

32 Y. Hu, Z. Zhang and C. Yang, Ultrason. Sonochem., 2008, 15, 665.

33 R. J. Wood, J., Lee and M. J. Bussemaker, Ultrasonics Sonochem., 2019, 58, 104645.

34 Q.-A. Zhang, Y. Shen, X.-H. Fan, J. F. Garcia Martin, X. Wang and Y. Song, Ultrason. Sonochem. 2015, 27, 96.

35 W. B. McNamara, Y. Didenko and K. S. Suslick, Nature, 1999, 401, 772.

36 K. Yasuda, T. Torii, K. Yasui, Y. liad, T. Tuziuti, M. Nakamura and Y. Asakura, Ultrason. Sonochem. 2007, 14, 699.

37 G. Chatel, Ultrason. Sonochem., 2018, 40, 117.

38 G. Cravotto, E. Borretto, M. Oliverio, A. Procopio and A. Penoni, Catal. Commun., 2015, 63, 2.

39 A. Tuulmets, S. Salmar and J. Järv, Sonochemistry in water organic solutions, Novinka Books, 2010, 54 p.

40 T. Lepoint and F. Lepoint-Mullie, In: Synthetic Organic Sonochemistry, ed. J.-L. Luche, Springer, Boston, MA, 1998.

41 G. H. Mahdavinia, S. Rostamizadeh, A. M. Amani and Z. Emdadi, Ultrason. Sonochem., 2009, 16, 7.

42 T. Deligeorgiev, S. Kaloyanova, N. Lesev and J. J. Vaquero, Ultrason. Sonochem., 2010, 17, 783.

43 A. Maleki, Ultrason. Sonochem., 2018, 40, 460. 
44 I. Mohammadpoor-Baltork, M. Moghadam, S. Tangestaninejad, V. Mirkhani and

Z. Eskandari, Ultras. Sonochem., 2010, 17, 857.

45 R. S. Disselkamp, Y.-H. Chin and C. H. F. Peden, J. Catal., 2004, 227, 552.

46 P. Qiu, B. Park, J. Choi, B. Thokchom, A. B. Pandit and J. Khim, Ultras. Sonochem., 2018, 45, 29.

47 N. Bremond, M. Arora, S. M. Dammer and D. Lohse, Phys. Fluids, 2006, 18, 121505.

48 D. E. Yount, E. W. Gillary and D. C. Hoffman, J. Acous. Soc. Amer., 1984, 76, 1511.

49 P. Gholami, A. Khataee, R. D. C. Soltani and A. Bhatnagar, Ultras. Sonochem., 2019, 58, 104681.

50Z. S. Kinzyabaeva and G. L. Sharipov, Ultras. Sonochem., 2018, 42, 119.

51 T. Ando, S. Sumi, T. Kawate, J. Ichihara and T. Hanafusa, J. Chem. Soc., Chem. Commun., 1984, 439.

52 J.-L. Luche, Ultras. Sonochem., 1996, 3, S215.

53 U. Neuenschwander, J. Neuenschwander and I. Hermans, Ultrason. Sonochem., 2012, 19, 1011.

54 R. Naik, A. Nizam, A. Siddekha and M. A Pasha, Ultrason. Sonochem., 2011, 18, 1124.

55 P. N. Amaniampong, A. Karam, Q. T. Trinh, K. Xu, H. Hirao, F. Jérôme and G. Chatel, Sci. Rep., 2017, 7, 40650. 
56 T. Cousin, G. Chatel, N. Kardos, B. Andrioletti and M. Draye, Ultrason. Sonochem., 2019, 53, 120.

57 P. Hu, Y. Ben-David and D. Milstein, J. Am. Chem. Soc., 2016, 138, 6143.

58 P. Contant, L. Forzy, U. Hengartner and G. Moine, Helv. Chim. Acta, 1990, 73, 1300.

59 W. Lijinsky, Cancer Res. 1974, 34, 255.

60 D. Lambert, Local Anesthetic Pharmacology, In: Stanley T.H., Ashburn M.A. (eds) Anesthesiology and Pain Management. Developments in Critical Care Medicine and Anesthesiology, Springer, 1994, Vol 29, 35.

61 S. Perrin, D. Montani, C. O'Connell, S. Günther, B. Girerd, L. Savale, C. Guignabert, O. Sitbon, G. Simonneau, M. Humbert and M.-C. Chaumais, Eur. Respir. J., 2015, 46, 1211.

62 R. Rothman and M. Baumann, Eur. J. Pharmacol., 2003, 479, 23.

63 L. Antkiewicz-Michaluk, I. Romanska, A. Wąsik and J. Michaluk, Neurotox. Res., 2017, 32, 94.

64 T. Kahl, K.-W. Schröder, F. Lawrence, W. Marshall, H. Höke and R. Jäckh, Aniline, In: Ullmann's Encyclopedia of Industrial Chemistry, Ed. Wiley-VCH, 2012.

65 S. M. Kelly and B. H. Lipshutz, Org. Lett., 2014, 16, 98.

66 M. Draye, G. Chatel and R. Duwald, Pharmaceuticals, 2020, 13, 1.

67 M. K. Basu, F. F. Becker and B. K. Banik, Tetrahedron Lett., 2000, 41, 5603. 
68 O. Elendu, M. Ojewumi, Y. D. Yeboah and E. E. Kalu, Int. J. Electrochem. Sci., 2015, 10, 10792.

69 ECHA, Sodium phosphinate, online: https://echa.europa.eu/fr/substanceinformation/-/substanceinfo/100.028.791 (accessed December 2020).

70 S. Letort, M. Lejeune, N. Kardos, E. Métay, F. Popowycz, M. Lemaire and M. Draye, Green Chem., 2017, 19, 4583.

71 G. Chen, Z. Liu, Y. Zhang, X. Shan, L. Jiang, Y. Zhao, W. He, Z. Feng, S. Yang and G. Liang, ACS Med. Chem. Lett., 2013, 4, 69.

72 M. Gaba, S. Singh and C. Mohan, Eur. J. Med. Chem., 2014, 76, 494.

73 N. Nongrum, G. K. Kharmawlong, J. W. S. Rani, N. Rahman, A. Dutta and R. Nongkhlaw, J. Heterocycl. Chem., 2019, 56, 2873.

74 S. Sarhandi, L. Zare Fekri and E. Vessally, Acta Chim. Slov., 2018, 65, 246.

75 M. Mohamed, J. Pharm. Sci., 2016, 53, 108.

76 B. H. Han and P. Boudjouk, J. Org. Chem., 1982, 47, 5030.

77 P. Boudjouk, D. P. Thompson, W. H. Ohrbom and B. Han, Organometallics, 1986, 5, 1257.

78 K. S. Suslick and S. J. Doktycz, J. Am. Chem. Soc., 1989, 111, 2342.

79 N. A. Ross and R. A. Bartsch, J. Heterocycl. Chem., 2001, 38, 1255.

80 N. A. Ross and R. A. Bartsch, J. Org. Chem. 2003, 68, 360.

81 R. G. Soengas and A. M. Estévez, Ultrason. Sonochem., 2012, 19, 916.

82 C.-J. Li, Tetrahedron, 1996, 52, 5643. 
83. S. M. Cenci, L. R. Cox and G. A. Leeke, ACS Sustainable Chem. Eng. 2014, 2, 1280.

84. B. D. Mather, K. Viswanathan and K. M. Miller, T. E. Long, Prog. Polym. Sci. 2006, 31, 487.

85. J. Christoffers, Eur. J. Org. Chem. 1998, 1998, 1259.

86. Y.-L Song, Y.-F. Dong, F. Wu, T. Yang and G.-L. Yang, Ultrason. Sonochem., 2015, 22, 119.

87. A. Koleva, N. Petkova and R. Nikolova, Synlett, 2016, 27, 2676.

88. B. Banerjee, Ultrason. Sonochem. 2017, 35, 1.

89. M. Draye and N. Kardos, Top. Curr. Chem., 2016, 374, 74.

90. P. R. Gogate, V. S. Sutkar and A. B. Pandit, Chem. Eng. J., 2011, 166, 1066.

91. V. S. Sutkar and P. R. Gogate, Chem. Eng. J., 2009, 155, 26.

92. J. W. K. Kambiz Farahmand, Exp. Heat Transfer, 2001, 14, 107.

93. Y. lida, K. Yasui, T. Tuziuti and M. Sivakumar, Microchem. J., 2005, 80, 159.

94. S. Merouani, O. Hamdaoui, Y. Rezgui and M. Guemini, Res. Chem. Intermediat., 2015, 41, 881.

95. K. Yasui, T. Kozuka, T. Tuziuti, A. Towata, Y. lida, J. King and P. Macey, Ultrason Sonochem., 2007, 14, 605.

96. N. Kerabchi, S. Merouani and O. Hamdaoui, Ultrason. Sonochem., 2018, 48, 136. 
97. N. Kerabchi, S. Merouani and O. Hamdaoui, Separ. Purif. Technol., 2018, 206, 118.

98. Y. T. Didenko and K. S. Suslick, Nature, 2002, 418, 394.

99. S. Koda, K. Tanaka, H. Sakamoto, T. Matsuoka and H. Nomura, J. Phys. Chem., 2004, 108, 11609.

100. A. A. Doinikov and S. T. Zavtrak, Physics Fluids, 1995, 7, 1923.

101. T. Tuziuti, K. Yasui, Y. lida, M. Sivakumar and S. Koda, J. Phys. Chem., 2004, $108,9011$.

102. R. A. Sheldon, Green Chem. 2007, 9, 1273.

103. R. A. Sheldon, ACS Sustainable Chem. Eng., 2018, 6, 32.

104. P. Tierce, Patent, 1998, FR2768948B1, WO1999016558A1.

105. S. I. Nikitenko, M. Brau, R. Pflieger, Ultrason. Sonochem., 2020, 67, 105189.

106. C. Cau, Y. Guari, T. Chave, J. Larionova, P. Pochon and S. I. Nikitenko, J. Phys. Chem., 2013, 117, 22827.

107. S. Balachandran, S. E. Kentish, R. Mawson and M. Ashokkumar, Ultrason. Sonochem., 2006, 13, 471.

108. E. S. Dassoff and Y. O. Li, Trends Food Sci. Technol., 2019, 86, 492.

109. G. Cravotto and P. Cintas, Chem. Eur. J., 2007, 13, 1902.

110. V. G. Gude, Resource-Efficient Technologies, 2015, 1, 116.

111. Y. Peng and G. Song, Green Chem., 2003, 5, 704. 
112 . D. Fernandez Rivas and S. Kuhn, Top. Curr. Chem., 2016, 374, 70.

113 . J. Sedelmeier, S. V. Ley, I. R. Baxendale and M. Baumann, Org. Lett., 2010, 12, 3618.

114. C. Delacour, D. Stephens, C. Lutz, R. Mettin and S. Kuhn, Org. Process Res. Dev., 2020, 24, 2085.

115. Y. Naruke, H. Tanaka and H. Harada, Electrochemistry, 2011, 79, 826.

116. G. Chatel and D. R. MacFarlane, Chem. Soc. Rev., 2014, 43, 8132.

117. G. Kaur, A. Sharma and B. Banerjee, ChemistrySelect, 2018, 3, 5283.

118. G. Chatel, R. Pflieger, E. Naffrechoux, S. I. Nikitenko, J. Suptil, C. Goux-Henry, N. Kardos, B. Andrioletti and M. Draye, ACS Sustainable Chem. Eng., 2013, 1, 137.

119. N. D. Oktaviyanti, Kartinin and A. Mun'im, Heliyon, 2019, 5, e02950.

120. D. Panda and S. Manickam, Ultrason. Sonochem., 2017, 36, 481.

121. Y. Son, M. Lim, J. Khim and M. Ashokkumar, Ind. Eng. Chem. Res., 2012, 51, 232.

122. R. Abazari, A. R. Mahjoub, S. Sanati, Z. Rezvani, Z. Hou and H. Dai, Inorg. Chem., 2019, 58, 1834.

123. S. Saito, Ultrasound Field and Bubbles, In: Sonochemistry and the Acoustic Bubble, Elsevier Inc., 2015, 11. 